\title{
Phantom bladder pain
}

\author{
Kyeong-Eon Park ${ }^{1}$, Kwang-Seong Cheon ${ }^{1}$, Seong-Ho Ok ${ }^{1}$, Young Ju Jeong ${ }^{2}$, and Heon Keun Lee \\ ${ }^{1}$ Department of Anesthesiology and Pain Medicine, Gyeongsang National University Hospital, Jinju, ${ }^{2}$ Chosun Nursing Collage, \\ Gwangju, Korea
}

A phantom syndrome is a pain syndrome that occurs when part of the body, such as the nose, tongue, breast, tooth, testis, penis, bladder, or anus, has been lost as the result of an accident or operation. Its frequency and etiology remain unclear [1]. Phantom bladder pain is a rare phantom syndrome that has not been reported previously in South Korea. This phantom urinary phenomenon occurs in patients with cystectomies, undergoing hemodialysis, or after spinal cord injury, in the forms of lower abdominal pain during urination, frequent urination, acute inconvenience with a full-bladder sensation, or urination with a sharp and burning pain [2].

Here, we report a patient who acquired phantom bladder pain 2 years after a cystectomy; pain was controlled successfully through a sympathetic ganglion block. A 59-year-old male patient presented with paroxysmal urgency and extreme pain accompanying bladder fullness and a bursting sensation that had started 1 month previously. He had undergone a cystectomy and prostatectomy, Miles operation, and ureteroileal conduit 2 years earlier due to rectal cancer. Defecation and urination were achieved through the connection of a colostomy and uretero-ileal conduit. A phantom sensation of the anus occurred a few months after the Miles operation, but without pain or any other inconvenience. Thus, the patient did not undergo any further treatment.

Owing to celiac metastasis with leakage, an ileo-colic anastomosis had been conducted 7 months before his visit to the pain clinic, and one month before his visit the patient had complained of bladder fullness and a bursting sensation with a desire to micturate and defecate. A spasmolytic agent, tiropramide $100 \mathrm{mg}$, was prescribed but resulted in no impro- vement. A psychiatric consultation revealed that the patient had clear consciousness without depression. The pain was increasing in frequency and strength, and the patient reported a continuous burning sensation with an intermittent sharp stinging sensation in the suprapubic region about 30 times per day. He scored the continuous pain as 6 on a visual analog scale (VAS), and he scored the intermittent paroxysmal pain that lasted $20-30 \mathrm{~min}$ as $9-10$. No aggravating or relieving factors were present, and no related symptoms were observed, except sleep disorders due to the pain. Gabapentin 1,200 mg/ $\mathrm{d}$, amitriptyline $10 \mathrm{mg} / \mathrm{d}$, oxycodone $40 \mathrm{mg} / \mathrm{d}$, and a fentanyl patch $50 \mu \mathrm{g} / \mathrm{h}$ were provided with no effect.

We injected $0.5 \%$ bupivacaine dextrose $6 \mathrm{mg}$ into the patient's L4/5 subarachnoid space, and required him to maintain a sitting position for $30 \mathrm{~min}$ to exclude a sympathetic ganglion blocking effect. The pain decreased to VAS 4 temporarily, then returned to the same level after $12 \mathrm{~h}$.

Then, a lumbar sympathetic ganglion block was conducted instead of a superior hypogastric plexus block. The needle was placed on the lower one-third of the L2 vertebral body using a $\mathrm{C}$-arm fluoroscope, then $0.5 \%$ bupivacaine $5 \mathrm{ml}$ was injected on the left, followed by confirmation of a warm sensation in the left foot. Then, the block was done on the right side, and the warm sensation was felt on the right side too. Because the VAS score diminished to 5, treatment with a neurolytic agent was advised, but lumbar sympathetic ganglion block at both the L2 and L3 levels was conducted two more times at the patient's request. The pain decreased to VAS 1-2, without recurrence, so the patient was discharged after 2 weeks.

Phantom limb pain is well-known, but other phantom pains

Corresponding author: Heon Keun Lee, M.D., Department of Anesthesiology and Pain Medicine, Gyeongsang National University Hospital, 92, Chilam-dong, Jinju 660-751, Korea. Tel: 82-55-750-8137, Fax: 82-55-750-8142, E-mail: ane1959@hanmail.net

(c) This is an open-access article distributed under the terms of the Creative Commons Attribution Non-Commercial License (http:// creativecommons.org/licenses/by-nc/3.0/), which permits unrestricted non-commercial use, distribution, and reproduction in any medium, provided the original work is properly cited. 
are rare. A phantom sensation and pain can occur in almost any organ, and is difficult to control despite the use of various drugs. A phantom sensation appears in $25 \%$ of patients 3 months after a mastectomy, and is consistent a year after the operation, and phantom pain appears in $13 \%$ of these patients. Additionally, $10-20 \%$ of patients report phantom pain of the anus after anal excision due to rectal cancer. However, phantom pain of the bladder is rarely reported. Brena and Sammons [2] reported phantom pain after cystectomy due to intractable cystitis, and the effects of lumbar sympathetic ganglion block. Fonseca et al. [3] reported phantom pain after metastatic bladder cancer operations.

The nerve system of the bladder is not fully understood, but is divided into a parasympathetic nerve system, starting from the sacral nerve and continuing to the pelvic nerve, and a sympathetic nerve system, from the lower thoracic and upper lumbar vertebrae via the hypogastric nerve. The bladder base is occupied by a terminal nerve of the hypogastric nerve, and the pelvic nerve is widely distributed throughout the bladder. The pelvic nerve, the A-delta, and the $\mathrm{C}$ fiber of the hypogastric nerve convey mechanosensitive reactions by sending urgency and pain signals when the bladder is expanded, not during bladder filling. The afferent fiber of the bladder changes according to bladder tissue status. For example, the bladder indicates urgency and pain during inflammation by overexcitement of afferent C fibers [4].

Generally, internal organs indicate extreme pain with strong expansion and contraction, and the pain is stronger with ischemia [4]. The sympathetic hypogastric nerve is important in the urgent and painful sensations of the pathological bladder. The effectiveness of sympathetic ganglion block has been reported in intractable interstitial cystitis that was not improved with repeated or continuous epidural block. Some reports indicate that sympathetic ganglion block is effective in deafferentation pain [5].

In this case, a saddle block was attempted to exclude effects of the sympathetic nerve, but it resulted in only temporary pain relief. Next, a sympathetic nerve block was planned with a lumbar sympathetic nerve block; this procedure resulted in marked pain relief, presumably because phantom limb pain is widely distributed deafferentation pain as a result of amputation of autonomic and somatic nerve systems, while internal organs have only autonomic nerve systems. This difference might also explain the remarkable result of the sympathetic nerve block. Because the nerve system of the bladder is not completely understood, the mechanosensitive reactions of the A-delta and $\mathrm{C}$ fibers require more study to clarify the neuronal mechanism(s) behind phantom bladder pain.

\section{Referneces}

1. Dorpat TL. Phantom sensation of internal organs. Compr Psychiatry 1971; 12: 27-35.

2. Brena SF, Sammons EE. Phantom urinary bladder pain: case report. Pain 1979; 7: 197-201.

3. Fonseca R, Frytak S, Mokri B. The case of the phantom bladder. J Urol 1997; 157: 1354.

4. Wyndaele JJ, De Wachter S. The basics behind bladder pain: a review of data on lower urinary tract sensation. Int J Urol 2003; 10 Suppl: S49-55.

5. Doi K, Saito Y, Nikai T, Morimoto N, Nakatani T, Sakura S. Lumbar sympathetic block for pain relief in two patients with interstitial cystitis. Reg Anesth Pain Med 2001; 26: 271-3. 